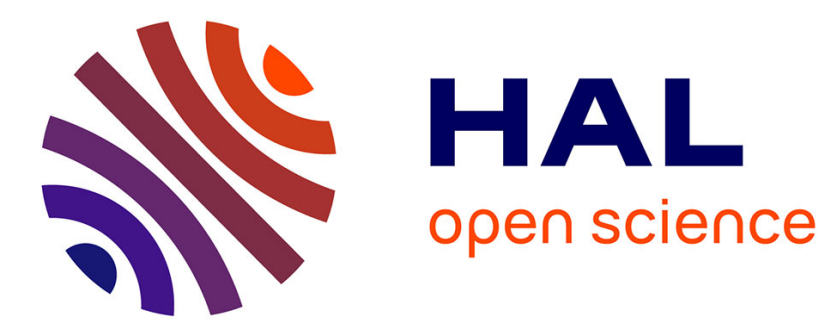

\title{
Design of a spatial database to analyze the forms and responsiveness of an urban environment using an ontological approach
}

Tolue Silavi, Farshad Hakimpour, Christophe Claramunt, Farshad Nourian

\section{- To cite this version:}

Tolue Silavi, Farshad Hakimpour, Christophe Claramunt, Farshad Nourian. Design of a spatial database to analyze the forms and responsiveness of an urban environment using an ontological approach. Cities, 2016, 52, pp.8-19. 10.1016/j.cities.2015.11.005 . hal-01527605

\section{HAL Id: hal-01527605 \\ https://hal.science/hal-01527605}

Submitted on 27 Sep 2017

HAL is a multi-disciplinary open access archive for the deposit and dissemination of scientific research documents, whether they are published or not. The documents may come from teaching and research institutions in France or abroad, or from public or private research centers.
L'archive ouverte pluridisciplinaire HAL, est destinée au dépôt et à la diffusion de documents scientifiques de niveau recherche, publiés ou non, émanant des établissements d'enseignement et de recherche français ou étrangers, des laboratoires publics ou privés. 


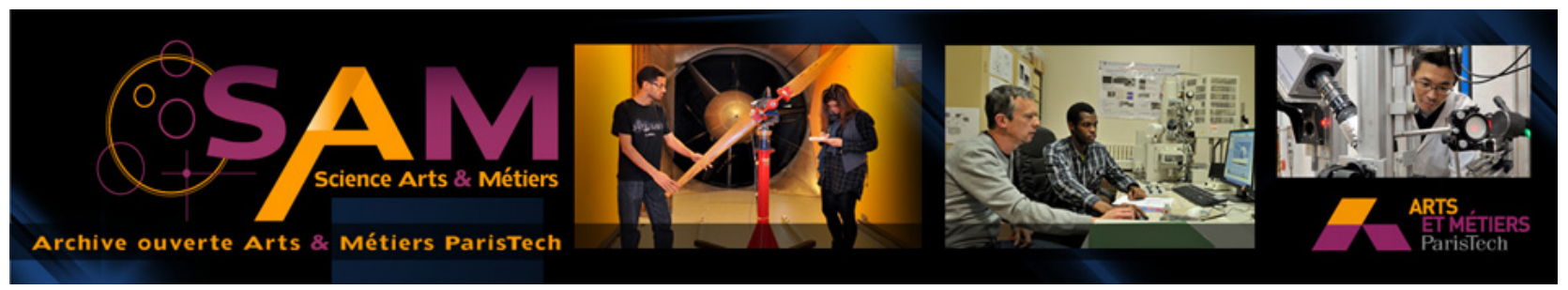

Science Arts \& Métiers (SAM)

is an open access repository that collects the work of Arts et Métiers ParisTech researchers and makes it freely available over the web where possible.

This is an author-deposited version published in: http://sam.ensam.eu Handle ID: .http://hdl.handle.net/null

\section{To cite this version :}

Tolue SILAVI, Farshad HAKIMPOUR, Christophe CLARAMUNT, Farshad NOURIAN - Design of a spatial database to analyze the forms and responsiveness of an urban environment using an ontological approach - Cities - Vol. 53, p.8-19 - 2016 


\title{
Design of a spatial database to analyze the forms and responsiveness of an urban environment using an ontological approach
}

\author{
Tolue Silavi ${ }^{a}$, Farshad Hakimpour ${ }^{\mathrm{a}}$, Christophe Claramunt ${ }^{\mathrm{b}}$, Farshad Nourian ${ }^{\mathrm{c}, *}$ \\ a Department of Geomatics, College of Engineering, University of Tehran, Iran \\ ${ }^{\mathrm{b}}$ Naval Academy Research Institute, France \\ c Department of Urban and Regional Planning, College of Fine Arts, University of Tehran, Iran
}

\begin{abstract}
A B S T R A C T
This paper introduces a spatial database and ontology-enabled framework that models and operationalizes the relation between urban forms and their responsiveness to the needs of its user. The objective is to offer a framework that provides a practical implementation of the concept of responsive environment introduced by Bentley, Alcock, Murrain, McGlynn and Smith (1985) in order to provide enough reusability and flexibility to reflect different urban modeling viewpoints and conceptualizations. The developed system is organized in three modules: the first and second modules model the ontology of a responsive environment and the layout of an urban environment while the third module is a spatial database that supports further computational analysis of urban forms. We applied several spatial data metrics that analyze the structural properties of urban forms and the emerging opportunities as identified by the notion of responsive environments. The framework is experimented and applied to the district 12, region 4 of the city of Tehran in Iran. The modeling abstractions lead to the generation of several semantic bridge rules and map layers that reflect different levels of urban responsiveness. The emerging patterns are qualitatively evaluated and found to be correlated with inhabitants' own perception of an urban environment. The results show that the suggested framework can be applied to the analysis of the responsive environments of urban forms.
\end{abstract}

\section{Introduction}

The identification of the properties that emerge from urban structures and their forms has been the main challenges of many urban studies (Futcher, Kershawb, \& Mills, 2013; SheikhMohammadZadeh \& Rajabi, 2013; Tomko \& Winter, 2013; Yimin, Li, Zheng, Guan, \& Liu, 2011). Such studies analyze the efficiency of the built environment in urban designs, development plans and executed policies. Urban forms, defined as the spatial arrangements of the city components, can be considered as a spatial layout that has a subtle relation with geometry. Notably, it appears that spatial databases as useful repositories of spatial data can be applied to the representation of urban forms. The main goal of this paper is to address and identify the most appropriate modeling abstractions, spatial database representations and manipulation operations that can reflect the complex properties of urban forms.

The premise of this research considers the concept of "responsive environments" as a critical property of urban environments. The objective behind the concept of "responsive environments" is to survey the opportunities offered by a given urban environment by taking into account different qualities of the city such as permeability, variety, legibility, robustness, visual appropriation, richness and personalization (Bentley, Alcock, Murrain, McGlynn, \& Smith, 1985). As defined by Bentley's group, responsive environments provide an innovative view for urban design. The focus of this paper is to analyze the state of responsiveness of a built environment as modeled by a spatial database of the layout.

The forms that appear in an urban environment can be perceived from different points of view, as well as related to several research domains because the city has been an object of many studies that range from social to economical and engineering sciences. In fact, any abstraction of an urban form is influenced by its context. This has a direct impact on the urban components identified by a modeling approach, their underlying properties, attributes and relations represented at the conceptual and logical database levels. These motivate the choice for an ontological framework that supports the representation of the urban morphology within a spatial database. Over the past few years, ontologies have been progressively established as privileged solution for formal representations of knowledge and specifications of a domain-based conceptualization (Gruber, 1993). When applied to 
an urban environment and the concept of responsiveness, many interrelated spatial concepts and properties, relations should be taken into account in order to infer logical consequences from represented facts. The implementation of such ontological representation within a spatial database approach is another issue addressed by this paper.

The research starts from the identification of the responsive environments and urban modeling abstractions in the layout of an urban environment. Those concepts are described by an ontology, and refined by additional properties and attributes. We retain the principle of a modular design procedure to favor flexibility and reusability of the developed components. The knowledge derived from the responsive environments, as along with the forms of an urban environment and the spatial database, are distributed in separated modules.

The rest of the paper is organized as follows. Section 2 briefly reviews related works and the main principles of our modeling approach. Section 3 introduces the modeling abstractions developed by our approach. Section 4 describes the implementation and application to the case study applied to the city of Tehran while finally Section 5 summarizes the paper and outlines future work.

\section{Modeling principles}

\subsection{Research background}

An important objective behind the study of urban forms is to conceptually and computationally explore the relationships between the forms and characteristics of the city (Bahrainy \& Khosravi, 2013; Chena, Li, Zheng, Guan, \& Liu, 2011; Jiang \& Claramunt, 2004a, 2004b; Khirfan, 2010; Tomko \& Winter, 2013). This also reflects one of the primary incentives behind the emergence of Space Syntax (Hillier \& Hanson, 1984) that, with its innovative computing perspective, made a significant and experimental contribution to the analysis of urban structures (Ariza-Villaverde, Jiménez-Hornero, \& Ravé, 2013; Jiang, Claramunt, \& Klarqvist, 2000; Jiang \& Liu, 2009; Koohsari, Kaczynski, Giles-Corti, \& Karakiewicz, 2013). Many recent researches have explored the characteristics of urban forms via an integration of spatial and semantics criteria for many application domains. Amongst several relevant examples, let us mention the analysis of urban climate (Eeftens et al., 2013; Futcher et al., 2013), disaster management (Liu, Luan, \& Zhong, 2012), energy consumption (Chena et al., 2011) and transportation studies (Gainza \& Livert, 2013; Rybarczyk \& Wu, 2014). A comprehensive review of these researches shows that the morphological criteria range significantly from first order to more intricate derived measures such as landscape metrics (Fan \& Myint, 2014; Herold, Couclelis, \& Clarke, 2005), densities and spatial distributions (Edussuriya, Chan, \& Yec, 2011; Filicaia, 2007), sky views and open spaces (Eeftens et al., 2013), and accessibility and connectivity measures (Contreras, Blaschke, Kienberger, \& Zeil, 2013; Eeftens et al., 2013). A few works have retained ontology-based approaches to model an urban system, and apply retrieval mechanisms to geographical information as suggested by (Lutz \& Klien, 2006). They used RACER (Haarslev \& Moller, 2004) as a terminological reasoning engine to find out which of the application concepts are equal to or subsumed by the query concept. Retrieval strategies are based on the exploration of a spatio-temporal ontology where these dimensions are considered as a sort of main filtering condition (Mata \& Claramunt, 2011). In our research, the semantic reasoning is performed based on the rules that establish the mathematical and spatial relations in the spatial database. Lüscher, Weibel, and Burghardt (2009) introduced a framework for an ontology-driven pattern recognition mechanism. In this research, the spatial structures of an urban concept is represented by an ontology, and mapped to measurable units in order to manipulate them in a spatial database. The concept that they consider in a spatial database is terraced houses. This concept has a simple and rigid definition in comparison to the concept responsive environment which is used in this research. Scheuer, Haase, and Meyer (2013) made a local knowledge accessible in form of a knowledge base and described the corresponding flood risk assessment ontology in order to put expert and local knowledge into operation. The terms and relations were gathered, and the relative importance of elicited terms was reviewed using Text-to-Onto. This is a tool for automatically extracting lexica from textual sources. Finally, the elicited concepts were matched against their semantic counterparts in the SWEET ontologies (Raskin \& Pan, 2005). The ontology matching performed by Scheuer et al. (2013) is a type of terminological matching from matching classes introduced by Delgado, Mart'inez-Gonzalez, and Finat (2013). In contrast, the ontology in our research establishes is a conceptual type (Delgado et al., 2013 ) in that different modeled perspectives of a domain are conceptually matched.

In another work, Bhatt, Hois, and Kutz (2011) stated that form, function and the relationship between the two serve a crucial role in design. However, a formal modelling of structural forms and resulting artefactual functions within design and design assistance systems remains elusive. They interpret "structural form" and "artefactual function" by specifying modular ontologies and their interplay for the architectural design domain. The modules are matched with the theory of $\varepsilon$-Connections (Kutz, Lutz, Wolter, \& Zakharyaschev, 2004) and have capabilities in talking about the link relations. In comparison to their work, our research deals with similar link relations that are implemented in the spatial database and are practically used to infer the instances of database.

\subsection{The architecture of the modeling approach}

One of the first objectives of this research was the identification of the urban form abstractions and modeling of the notion of responsive environment. This leads us to retain an ontological approach. Ontologies, as "explicit and formalized specifications of conceptualizations" (Gruber, 1993), play an important role to extract and formalize concepts. Guarino (1998) refines Gruber's definition by making clear the difference between an ontology and a conceptualization: An ontology is a logical theory accounting for the intended meaning of a formal vocabulary. More precisely, an ontology provides a conceptualization by (i) identifying the relevant terms that form the concept for the domain considered and (ii) devising semantic relations and axioms to account for their intended meaning (Scheuer et al., 2013). Regarding the context of an urban system, the extent of available perceptions ranges from inhabitants to stakeholders and urban designers with various expertise and interests. The objective of an ontology approach is to provide a common reference, then avoiding conceptual conflicts at the modeling level (Jung, Sun, \& Yuan, 2013; Kotoulas et al., 2014). Next, these ontologies should be mapped appropriately towards a spatial database. Such a spatial database models urban forms and structural properties as spatial data types and provides the city's skeleton and urban layout. The modeling approach is designed as a system distributed in three modules that comprise the ontology of the urban subject, the ontology of the urban environment and an urban spatial database (Fig. 1). Ontology modularization is a technique that favors reusability and flexibility which is one of the valuable properties of ontologies (Uschold \& Gruninger, 1996). The modularization favors reusability when applying the concept of responsive environment in to a given urban layout. These modules intercommunicate with each other. The main objective of these communication components, called bridges, is to map the semantics from one module to another module. Each so called bridge is formally defined by a set of semantic rules that support the semantic mapping processes. At last a semantic reasoning that technically denotes a combination of subsumption and instantiation reasoning is performed. The subsumption reasoning infers the subclass, superclass and equal type relations between concepts and instantiation reasoning infers the instances that belong to a concept. 


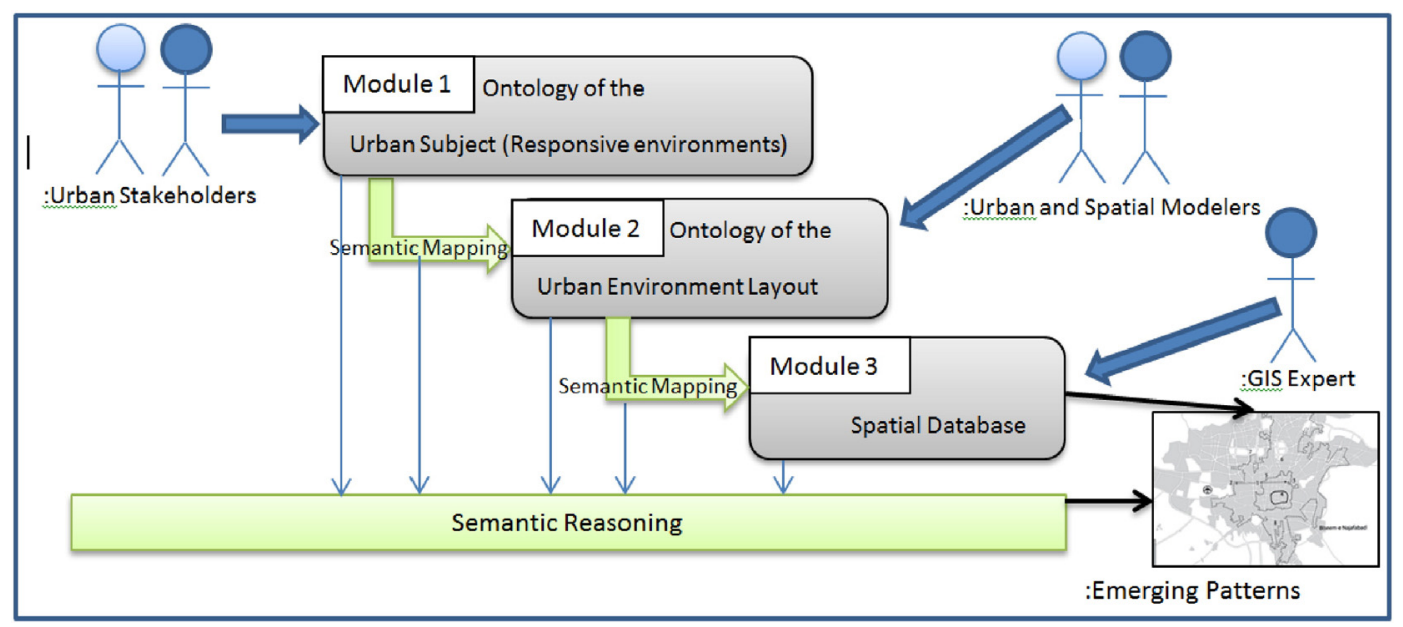

Fig. 1. The architecture of the modeling approach.

\section{Model development}

The modeling efforts developed at the formal level have been fully implemented for each module described in Fig. 1. The ontologies developed are specified by the Protégé Ontology Editor (version 4.1 beta) (Noy, Fergerson, \& Musen, 2000). The ontologies introduced in the first and second modules are formally described by $\mathrm{OWL}^{1}$ and mapped to $\mathrm{RDF}^{2}$ graphs that are fully supported by Protégé. RDF data are transferred to the database as a data type named $\mathrm{N}$-triple. A N-triple is a plain text line-based serialization format supported by RDF. A sample of a statement in N-triple is given below. It describes the Class2 from module M1which is also a subclass of Class2 from module M1:

$($ '<M1:Class2>', '<rdfs: subClassOf $>$ ', '<M1:Class1>')

The urban spatial database and the mapping between the different modules have been created and populated in Oracle ${ }^{\circledR}$ while a series of spatial attributes are implemented on top of ArcGIS. Finally, the cartographical outputs have been performed under Map Viewer connected to the database and can be visualized using Web Map Services (WMS).

\subsection{Responsive environments}

The notion of "Responsive environments" arises from the idea that "the built environment should provide its users with some flexible setting, enriching their opportunities by maximizing degree of choice available to them” (Bentley et al., 1985). Bentley et al. parameterized their idea via seven levels:

1. Permeability: where people can and where they cannot go.

2. Variety: the range of usages that a place provides.

3. Legibility: the ease with which people can understand the spatial layout of a place.

4. Robustness: the degree to which people can use a given place for different purposes and activities.

5. Visual Appropriateness: the way in which the design can physically make people aware of the choices the place provides.

6. Richness: ways to increase the choice of sense experience that users can enjoy (i.e., experiences of touch, sound, light, and so on and so forth).

7. Personalization: designs that encourage people to put their own stamp on their places.

These qualities should be ideally taken into account for the design of buildings and outdoor spaces, as well as means for the evaluation of the opportunities offered to inhabitants by existing buildings and outdoor places. This research is specifically oriented to the latter objective. Therefore, an initial step of the modeling approach is oriented to the identification of an ontology and formal representation of the different dimensions, mentioned above, of a responsive environment. Fig. 2 comprehensively shows the qualities identified for the responsive environments. Let us mention that all the responsive environment dimensions are the ones introduced by Bentley et al. (1985). They are all also characterized by Bentley et al. with the exception of "legibility" introduced in early work by Lynch (1960). Furthermore, some of the dimensions introduced by Bentley's group such as Personalization and Visual Appropriateness, as well as other sub-criteria, are not considered as they do not explicitly appear in the layout of an urban environment.

The hierarchy in Fig. 2 is a conceptual diagram that acts as a reference for the development of the ontology of "Responsive Environments" as implemented in Protégé. The below statements are samples of such ontology that declares the classes ResponsiveEnvironment, PermeableEnvironment

\footnotetext{
1 Web Ontology Language.

2 Resource Description Framework.
} 

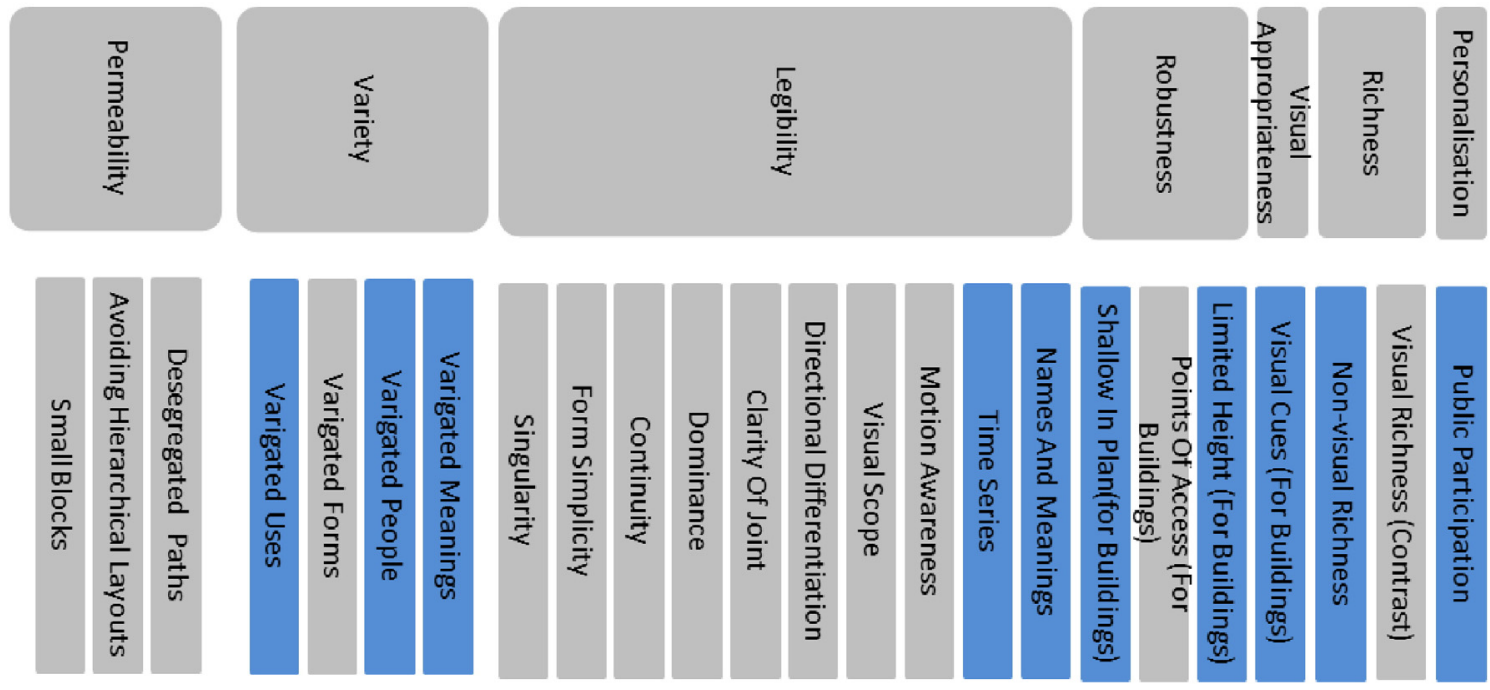

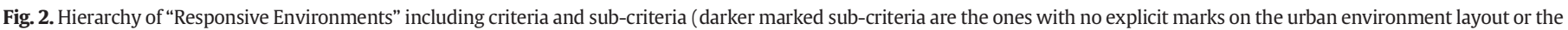

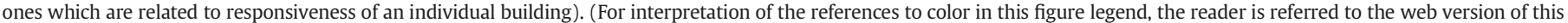
article.)

and EnvironmentsWithDesegregatedPath and the subclass relations between them. The last two statements denote that a Responsive Environment is subclass of a PermeableEnvironment and a PermeableEnvironment is subclass of an EnvironmentsWithDesegregatedPath.

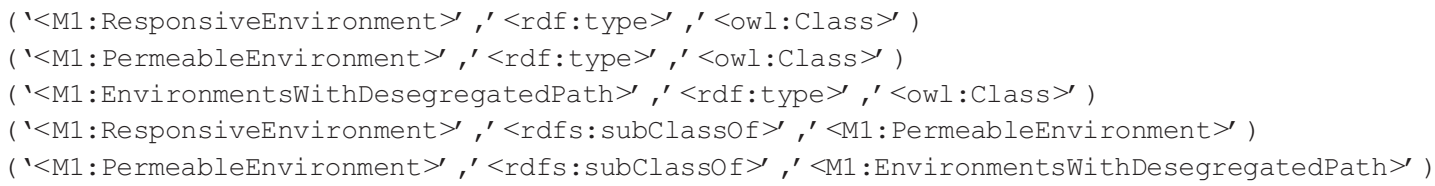

\subsection{Urban layout modeling}

According to Lynch (1960), human spatial conceptualizations of cities are made of spatial object representations that can be considered as instances of five elements (i.e., paths, edges, districts, nodes, landmarks). In order to conceptualize the layout of an urban environment, Lynch's classification is applied and refined to a lower level of abstraction, as illustrated ontologically and graphically in Fig. 3. For instance, the element Parcel is explicit in the layout and is one of the structural abstractions represented. Each Parcel has a parcel boundary and one to many building lots. A ParcelsSet is an aggregation of Parcels. A ParcelsSet might be either a set of Connected Parcels or a set of Separated Parcels. A set of ConnectedParcels denotes a set of connected Parcels as follows: two parcels are connected when they share a boundary, then the graph derived from this relation is a connected graph. When the later property is not fulfilled, then the ParcelsSet is denoted as Separated Parcels. When the parcels of ConnectedParcels have also connections to parcels exterior to the set (Fig. $3 \mathrm{j}$ ) then the ConnectedParcels is named as ParcelsWithConnections and it is IsolatedParcels in the contrary. Moreover, a set of IsolatedParcels is also named Block. A Block is materialized by a set of connected parcels that are isolated by the paths surrounding them. An UrbanArea is a bounded space of a city with all paths, edges, parcels and blocks which completely lay in. A District in this research denotes a set of parcels without any other urban component and is equivalent to a Parcel Set. In this research the districts are the parts consisting of parcels on two sides of the paths, unlike the blocks that are on one side of the surrounded paths. Paths are elements that people observe in the city while moving through. Finally, other environmental elements are arranged and interrelated according to the semantics that emerge at different levels of abstraction as suggested by Tomko and Winter (2013). Edges are boundaries between two different phases and contexts (Tomko \& Winter, 2013) that in the layouts the outer boundaries of blocks are the most important edges. Nodes are strategic spots in the streets layout (Tomko \& Winter, 2013). Landmarks are another type of spotted and interpreted elements whose importance is often user dependent. However, landmarks are often interpreted from existing objects in the layout and not always directly represented from the abstractions mentioned above so they are not considered by the modeling approach. All these components are not applied to the analysis of responsive environments but for the sake of generality and reusability of the introduced model we keep most of the components. This enables this model to analyze additional urban form properties in future research.

Overall, the urban layout components can be quantified by spatial and structural metrics. The metrics considered here are spatial metrics that are defined as landscape metrics (Aguilera, Valenzuelaa, \& Botequilha-Leitãob, 2011; McGarigal \& Marks, 1995). They are complemented by a series of additional measures that qualify directions, lengths, widths, bends and symmetries of paths and also continuity and alignment for edges. Landscape metrics fall into two general categories: those that quantify diverse patch types in and those that quantify the spatial configuration of a given urban layout (Gustafson, 1998). There has been an increasing interest in applying the second category to urban studies (Aguilera et al., 2011; Herold et al., 2005; Voordea, Jacquet, \& Cantersa, 2011). 

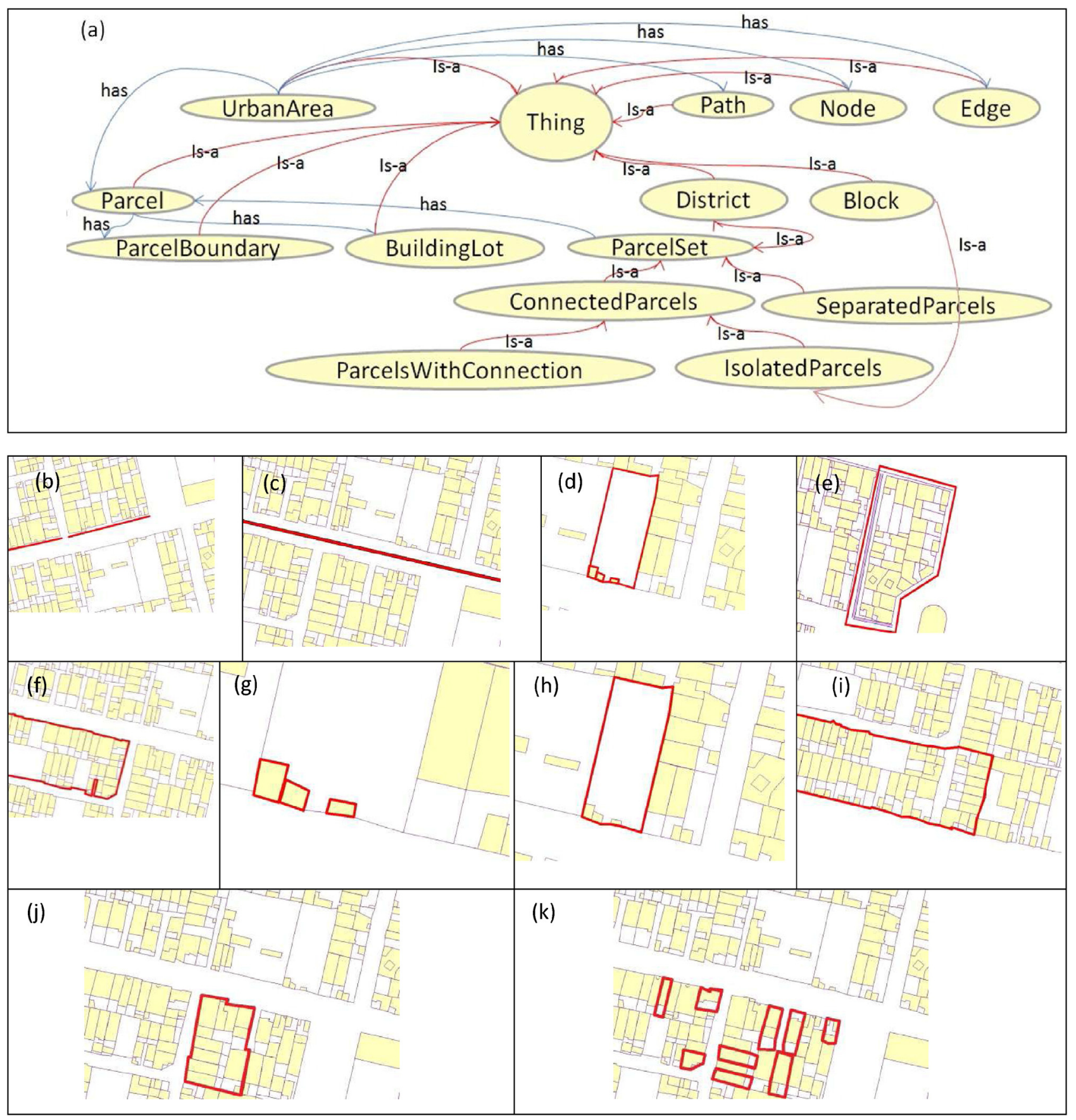

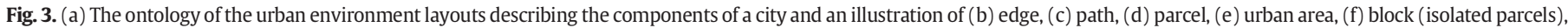
(g) building lot, (h) parcel boundary, (i) district (parcel set), (j) parcel set (parcels with connections to others) and (k) parcel set (separated parcels).

At the ontological level, all the components are specified by the constructors $\mathrm{Cl}$ ass and SubclassOf. All the selected spatial metrics are specified in the developed ontology as Datat ypeProperty to the appropriate classes. Thanks to the flexibility and reusability of the ontological model developed, this set of metrics can be extended. In addition to these metric properties, the logical relationships between the components are specified by objectProperties. For example, "the path hasIntersection only path". This statement means that each path can have some intersections with other paths only and not with other components. These properties are specified within Protégé as a hierarchy of classes and super-classes and by the module "urban environment" as illustrated in Fig. 1. For example, the statements below denote a class Block, equivalency of Block to IsolatetParcels, a data type property hasArea with a float type for Block:

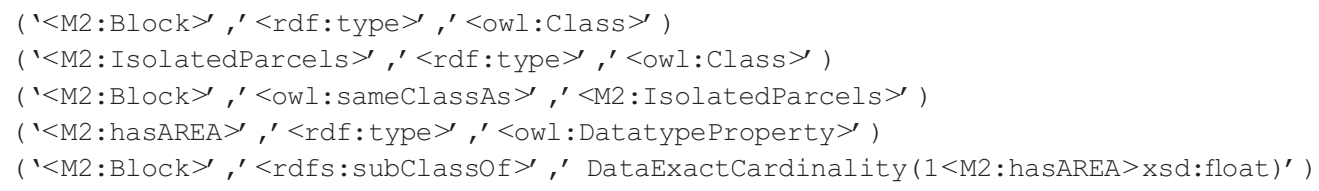


Table 1

The bridge rules from the ontology of "responsive environments" to the ontology of "urban environments".

\begin{tabular}{|c|c|}
\hline Rule no. & Bridge rules \\
\hline 1 & The Environment has Small Blocks if $\forall$ Block $\in$ Environment $\Longrightarrow$ AREA(Block) $\in$ LowAREA \\
\hline 2 & The Environment isn't Hierarchical if $\forall$ Block $\in$ Environment $\Longrightarrow \operatorname{PARA}($ Block $) \in$ LowPARA $\wedge$ NCA (Block) $\in$ LowNCA \\
\hline 3 & The Environment is less affected by Segregation if $\forall$ Path $\in$ Environment $\Longrightarrow$ WIDTH(Path) $\in$ HighWIDTH \\
\hline 4 & The Environment is Variegated if $\forall$ District $\in$ Environment $\Longrightarrow$ AREA_SD(District) $\in$ HighAREA_SD $\wedge$ SHAPE_SD(District) $\in$ HighSHAPE_SD \\
\hline 5 & $\begin{array}{l}\text { The Environment has Clear Joins if } \forall \text { Path1 } \in \text { Environment } \Longrightarrow \neg \exists \text { Path2 } \in \text { Environment [Intersection(Path1,Path2)] } \vee \exists \text { Path2 } \in \text { Environment } \\
{\left[70^{\circ}<\text { IntersectionAngle(Path1,Path2) }<110^{\circ}\right]}\end{array}$ \\
\hline 6 & The Environment has Continuance if $\forall$ Edge $\in$ Environment $\Longrightarrow$ Continuance(Edge) $\in$ LowContinuance \\
\hline 7 & The Environment has Directional Differentiation if $\forall$ Path $\in$ Environment $\Longrightarrow$ Symmetry(Path) $\in$ Low Symmetry \\
\hline 8 & The Environment has Domination if $\forall$ District $\in$ Environment $\Longrightarrow$ AREA_SD(District) $\in$ LowAREA_SD $\wedge$ AREA_RA(District) $\in$ HighAREA_RA \\
\hline 9 & The Environment has Motion Awareness if $\forall$ Path $\in$ Environment $\Longrightarrow$ Bend(Path) $\in$ HighBend \\
\hline 10 & The Environment has Simplicity if $\forall$ ParcelBoundary $\in$ Environment $\Longrightarrow$ SHAPE(ParcelBoundary) $\in$ LowSHAPE \\
\hline 11 & The Environment has Singularity if $\forall$ District $\in$ Environment $\Longrightarrow$ SHAPE_SD(District) $\in$ HighSHAPE_SD \\
\hline 12 & The Environment has Visual Scope if $\forall$ Edge $\in$ Environment $\Longrightarrow$ ALIGNMENT(Edge) $\in$ HighALIGNMENT \\
\hline 13 & The Environment has Visual Scope if $\forall$ Path $\in$ Environment $\Longrightarrow$ Bend(Path) $\in$ LowBend \\
\hline 14 & The Environment is Robust if $\forall$ ParcelBoundary $\in$ Environment $\Longrightarrow$ ECON(parcelBoundary) $\in$ HighECON \\
\hline 15 & The Environment is Robust if $\forall$ Block $\in$ Environment $\Longrightarrow$ PARA(Block) $\in$ HighPARA \\
\hline 16 & The Environment has Visual Rich Sense if $\forall$ District $\in$ Environment $\Longrightarrow$ ARAE_SD(District) $\in$ HighAREA_SD \\
\hline
\end{tabular}

\subsection{Spatial database module}

The representation of the urban layout forms is based on the model introduced in the previous sections, it approximated via parcels and paths. The parcels are the basic components of the spatial database of an urban layout. Paths are materialized from streets and other corridors between blocks. The other city components include blocks, districts, edges and many others spatially inferred from parcels and paths as stated in Section 3.2. When considering the ontology of an urban environment as developed in the previous section, each measurable attribute of a given city component can be derived and instantiated in the spatial database. All these attributes are spatially computable using appropriate commands, functions and tools on top of the spatial database. They are stored in a tabular structure that for each component measure there is a specific data field. In order to successfully implement the spatial database and preserve the integrity of the whole system, such data have been converted to N-triple data types. The statements below are two samples that denote the values of width and PARA (perimeter to area ratio, one of the landscape metrics) for path1792 and block259, respectively, after conversion to N-Triples. Path1792 and block259 are two sample instances of Path and Block.

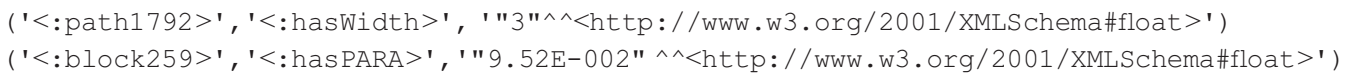

\subsection{Bridges between modules}

The previous sections introduced different modules, from the ontological to the database levels, and from the "responsive environments" to the "urban environments", as well as the representation of the spatial features and their attributes. As illustrated in Fig. 1, bridges are connector components between modules and that are mandatory to settle the system. These bridges are defined as rule bases in the database. Two categories of rules have been defined, that is, "external rule base" and "internal rule base". The external rule base matches the definition of the responsive environments to its respective ontological representation of an urban component. As illustrated in Fig. 2, 14 determinants for a "Responsive environment" in relation to the forms of an urban environment are identified. This leads to 16 detailed rules that qualify the components of an urban environment layout. These rules are structurally implemented in an "external rule base". Table 1 shows the designed bridge rules as modeled in predicate logic. The main role of these rules is to give a spatial interpretation of the qualities of a responsive environment and to specify these parameters according to the properties of an urban layout. These rules model such parameters using the geometrical characteristics of the urban components. The semantic and geometric properties behind bridge rules are described below:

- Small blocks: a block with a small area (Rule 1).

- Avoiding hierarchical Layouts: a permeable hierarchy denotes a street network with fewer cul-de-sacs and dead ends. This leads to blocks with fewer notches in their perimeter and thus small perimeter to area ratio (PARA from landscape metrics) and a few number of disjoint core areas (NCA from landscape metrics) (Rule 2). The NCA is computed by counting the disjoint polygons made by a buffer with a specified negative distance in. Fig. 4 presents a comparative view for a block in a hierarchical network and the same block when the notches are removed.

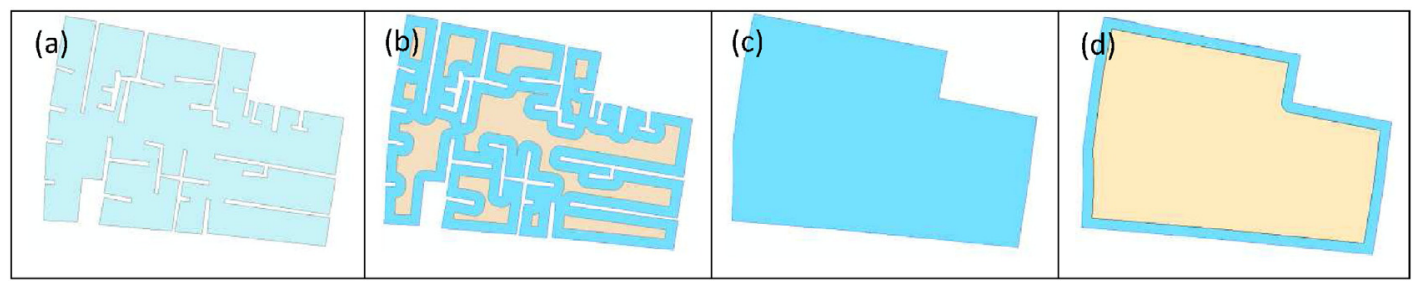

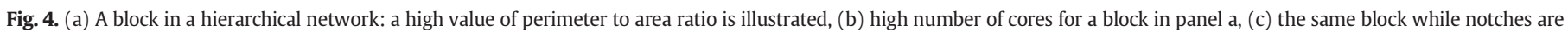
removed: lower value of perimeter to area ratio is illustrated and (d) only one core instead of many cores in panel b. 


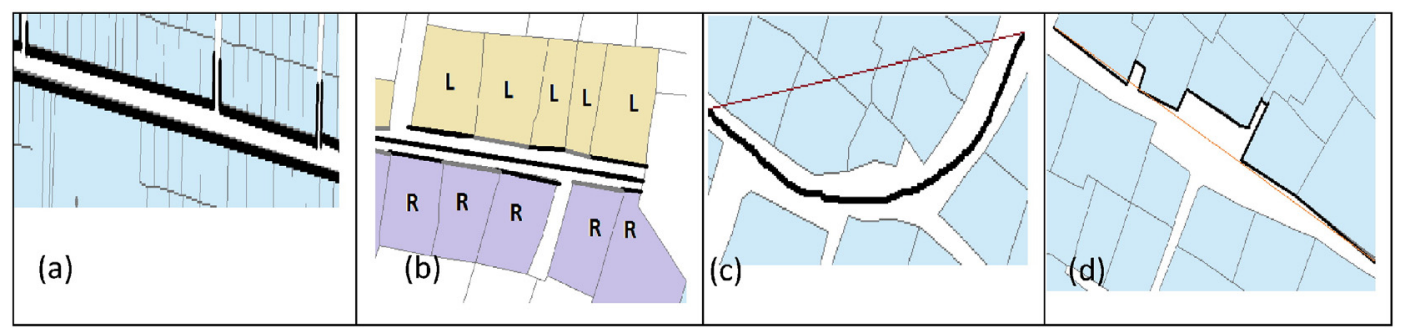

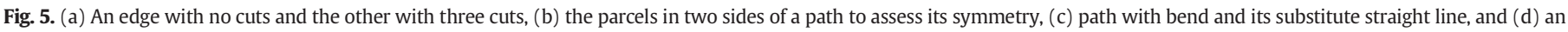
unaligned edge and its substitute straight line.

- Desegregated paths: paths wide enough are less likely to be affected by segregation (Rule 3).

- Variegated forms: the standard deviation of the parcel's area and parcel's SHAPE index in a given district can measure the degree of variegation (Rule 4). The SHAPE index is one of the landscape metrics that measures the complexity of patch shape compared to a standard shape (square) of the same size. The shape index is given as SHAPE $=0.25 \mathrm{p} / \sqrt{\mathrm{a}}$. The parameters $\mathrm{p}$ and a are the perimeter and the area of a parcel respectively.

- Clear joins: clarity of a street junction means enough distinguishability of intersections by the intersection angle. Such angle should be higher than 70 degrees and less than 110 degrees as suggested in a related work (TAC, 1999) (Rule 5).

- Continuance: An edge with a very few notches and cuts gives a sense of continuance. The most important edges in an urban layout are the outer boundary of blocks. This is computed by counting the paths that cut an edge (Rule 6). Fig. 5.a presents an edge with no cuts and a second one with 3 cuts along itself.

- Directional differentiation: Paths with symmetric sides give no sense of direction to passengers (Rule 7). The symmetry of a path relatively depends on street view differences between the two sides and can be approximated by the street layouts to some extent. It is done by differentiating the parcels in each side of paths, their numbers, the average of their areas and the average of their edges along each side. These three parameters after normalization can be averaged to give a quantity of directional differentiation for each path. Fig. $5 \mathrm{~b}$ presents the parcels on two sides of a path. Their edges along the path are colored black and gray.

- Dominance: The dominance reflects some conspicuous parcels in a given extent. It is modeled in the layout for a district where some parcels have an area largely higher than the others (Rule 8). If the standard deviation of the areas of a large set of parcels is relatively small while the range between minimum and maximum is relatively large, this means that some parcels dominate the others according to their large size.

- Motion awareness: The feeling of motion in a path is less perceived in straight paths, as bends in a path can improve motion awareness. For each path, the difference between its length and the straight Euclidean distance from its start to its end point gives an evaluation of bendiness (Fig. 5c) (Rule 9).

- Simplicity: Simplicity reflects shapes that generally fit more to a simple shape such as a square. The SHAPE index of parcel boundaries is used to quantify the simplicity (Rule 10).

- Singularity: The notion of singularity denotes the contrast and distinguishability of some objects in one scene. If the standard deviation of the parcels' SHAPE in a given layout is high enough, they can model the singularity of parcels as well.

- Visual Scope: Unaligned edges (Fig. 5d (Rule 12) as well as path curvature (Fig. 5c) (Rule 13) limit the visual scope.

- Points of Access: The accessibility to a parcel increases its links to the open spaces. The edge contrast index from landscape metrics (ECON) as calculated in equation 3 can quantify it (Rule 14). It is given as ECON $=\mathrm{p}_{\mathrm{k}} / \mathrm{p}$. The parameter $p_{k}$ denotes the length of a given parcel that is adjacent to open spaces while $p$ is the perimeter of the parcel. Also blocks with a higher perimeter to area ratio (PARA from landscape metrics) generally satisfy higher links between their parcels to open spaces (Rule 15).

- Visual Richness (Contrast): Standard deviation is a custom quantifier for contrast as defined in (Moulden, Kingdom, \& Gatley, 1990), while the visual contrast is quantified by a standard deviation of parcels' area in the layout (Rule 16).

Technically, each rule is specified with three parts: antecedents, filters that are optional and consequents. All the 16 rules introduced in Table 2 have been inserter to "external rule base" in the database. The components of rule 2 as a sample are illustrated in Table 2. M1, M2 and M3 refer to namespaces of first, second and spatial database modules subsequently.

An internal rule base establishes a semantic bridge between the triples specified in the spatial database module (introduced in Section 3.3) to the triples identified by the ontology of an urban environment (introduced in Section 3.2). For instance, the concepts that model the lowness, highness and related spatial metrics and break points are inserted into the system by this bridge. Table 3 presents some examples of the rules inserted in the internal rule base. For example the first rule says that if $x$ is path and $y$ is its width and $y$ is greater than a given value (e.g., given to 7.07 in this example derived from the case study) then the width of $x$ is set to "High". The 7.07, 891.77 and 1.08 are the means of WIDTH, AREA_SD and SHAPE in the extent that will be more discussed in the next section.

Table 2

Rule example defined for permeable hierarchies as inserted in the external rule base. The rule antecedents satisfy the conditions defined in Table 1.

\begin{tabular}{ll}
\hline Antecedent & Consequent \\
\hline (? x rdf: type M2:Block) & (?xM1: has M1: EmvironmentsWithPermeableHierarchies) \\
$($ ? $\mathrm{M} 2:$ hasPARA $:$ LOW) & \\
$($ ( x M2 : hasNCA $:$ LOW) & \\
\hline
\end{tabular}


Table 3

Some example rules of the internal rule base.

\begin{tabular}{|c|c|c|}
\hline Antecedent & Filter & Consequent \\
\hline $\begin{array}{l}(? \times \text { rdf }: \text { type M3 : Path) } \\
(? \times \text { M3 }: \text { hasWidth ?y) }\end{array}$ & $y>7.07$ & (? x M2: hasWidth M2: High) \\
\hline $\begin{array}{l}\text { (?x rdf: type M3:District) } \\
\text { (?x M3 :hasAREA_SD?y) }\end{array}$ & $y>891.77$ & (? x M2 : hasAREA_SD M2:High) \\
\hline $\begin{array}{l}\text { (? x rdf: type : ParcelBoundary) } \\
\text { (? x M3: hasSHAPE?y) }\end{array}$ & $y<1.08$ & (? x M2: has SHAPE M2: Low) \\
\hline
\end{tabular}

\section{Implementation}

Tehran, the capital of Iran, with an area of about $686.3 \mathrm{~km}^{2}$ is distributed in 22 municipal districts and 134 regions. The experimental evaluation of this research is conducted with a study area related to district 12, region 4 (Fig. 6), located in the central part of Tehran and containing diverse interrelated land uses. This region is about $3 \mathrm{~km}^{2}$ and is known because of crowded bazaar. In the history of Tehran, some districts such as district 12 have been developed without any approved plan that apparently leads to ill-structured urban forms. Such characteristics reduce life desirability and responsiveness of these environments.

\subsection{Experimental results}

The final step is to match an urban area to the identified properties of the responsive environment. It is performed by a specification of the semantic queries in the database, applied to N-triple terms, either existed or inferred from the rule bases previously defined. Since the selected study area is inherently unstructured from an urban design point of view and also because of lack of a standard or previous work oriented to the modeling of the urban layouts, satisfying all the identified parameters is a non-straightforward task. Therefore, the mean values of the quantitative parameters exhibited in the extent of the study area are set as thresholds to relatively determine the lowness and highness of parameters (e.g., the values 7.07, 891.77 and 1.08 that appear in Table 3 give the means of the parameters WIDTH, AREA_SD and SHAPE, respectively). The application of our modeling framework provides a representation of the concept of responsive environment in the selected study extent, and a practical modeling approach to be confronted to the perception and experience of the people that live and work in the study area.

The matching procedure is applied to the 16 identified properties separately (Fig. 7). The cartographical outputs presented below have been generated by a Map Viewer connected to the spatial database. The parcels and path data inputs used for this research are given by the cadastral maps at the scale of 1:2000. The other components are derived according to their spatial definition as introduced in Sections 3.2 and 3.3. The extent is divided into about 70 equal area districts to be analyzed by district-related rules. Each map layer presents the instances of an urban layout component that satisfy the condition of the related rule in black while the unsatisfied ones are shown by a light gray. The patterns reveal structural properties that can be interpreted either individually, compared to other patterns, or aggregated to reveal more comprehensive properties.

In some limited cases, and this is due to the multiplicity of parameters and the high number of parameters, some contradictory patterns might arises as for Fig. 7.4 and Fig. 7.8. The variegated environments are described as environments with diversity of their parcels' area (i.e., derived by the value of the standard deviation of parcels' area, the higher the standard deviation the higher the diversity). On the other hand, the parameter "domination" is related to the emergence of many similar parcels regarding their areas and a few large ones (i.e., low standard deviation but high range). There is a similar condition for paths that satisfy motion awareness (Fig. 7.9) and the ones that satisfy the visual scopes (Fig. 7.13). Therefore it appears that a small area around the "Harandi" street's green area (illustrated by an oval in Fig. 8) emerges as the most responsive environment of the study area. This extent has positive signs in the patterns of all the rules except the rules of dominance (Fig. 7.8) and motion awareness (Fig. 7.9).

\subsection{Experimental evaluation}

This section qualitatively evaluates the results exhibited by the responsive environment parameters shown in the respective map layers that emerge in Fig. 7. The objective is to compare the patterns revealed by the approach to the inhabitants' own perception of their urban environment and other evidences. In order to assess the permeability of the study area, a questionnaire complemented by appropriate instructions and definition of the notion of "permeability" were distributed to a panel of inhabitants, who work, live or study in the selected area. A map of the study area with 29 dotted important places together with their address and a table to fill the level of permeability were given to the panelists to express their opinion. The questionnaires were answered by 50 residents in the study area with permeability expressed in the range between 1 and 3 for high, moderate and less permeability respectively. Fig. 8 locates the requested locations numbered 1 to 29 . Another questionnaire was also prepared to evaluate the parameter "legibility". The legibility parameter were evaluated in predefined neighborhoods labeled from " $a$ " to " $i$ " (Fig. 8). The graphs in Figs. 9 and 10 present the points and neighborhoods that the permeability and legibility have been evaluated respectively. The degrees shown for each location are the mean of grades obtained from 50 questionnaires.

While Fig. 7 shows the most responsive areas that materialize from the 'permeability' and 'legibility" parameters, Fig. 11 generates a continuous spatial representation of these two parameters derived from the mean of the opinions expressed. Regarding the permeability patterns revealed by Fig. 11.a, it clearly shows that the four most permeable neighborhoods are located close and around the streets "Nasiri", "Hatami Brothers", "Maleki" and "Ba Atefe pour" (the dark regions that appear in Fig. 11.a). When compared to the emerging patterns of the responsive environments shown in Fig. 7.1 to Fig. 7.3, the role of the street "Nasiri" and "Maleki" are confirmed by the patterns that emerge in Fig. 7.1, while the permeability of the street "Hatami

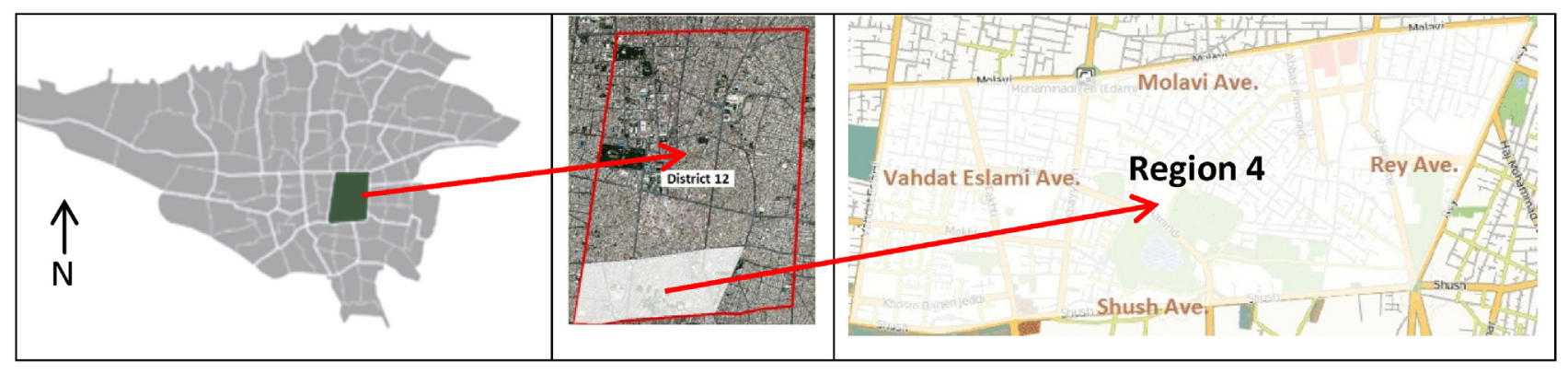

Fig. 6. Location of District 12 and its 4th region on Tehran map. 


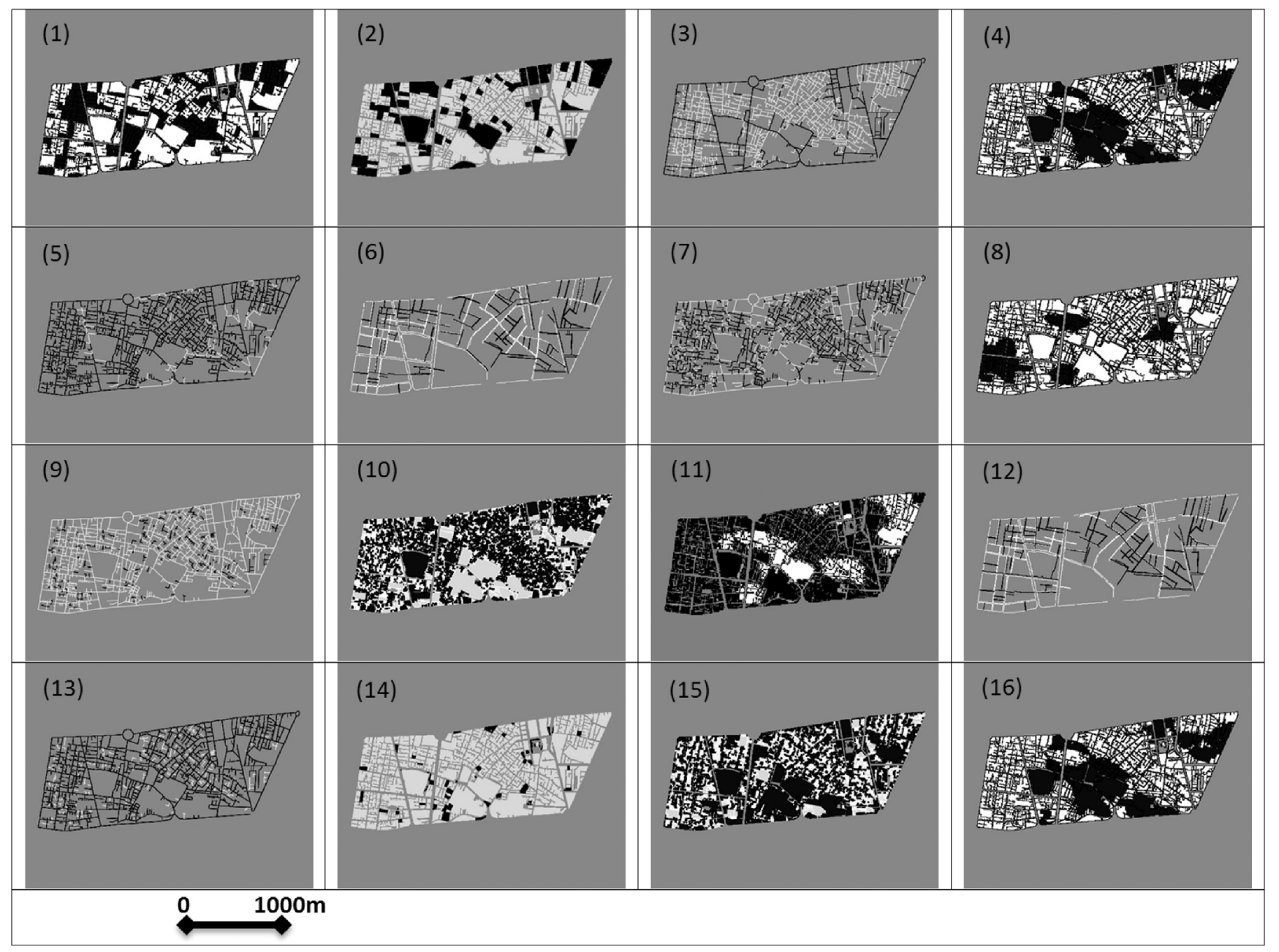

Fig. 7. Map layers related to bridge rules 1 to 16 as described in Table 1 .

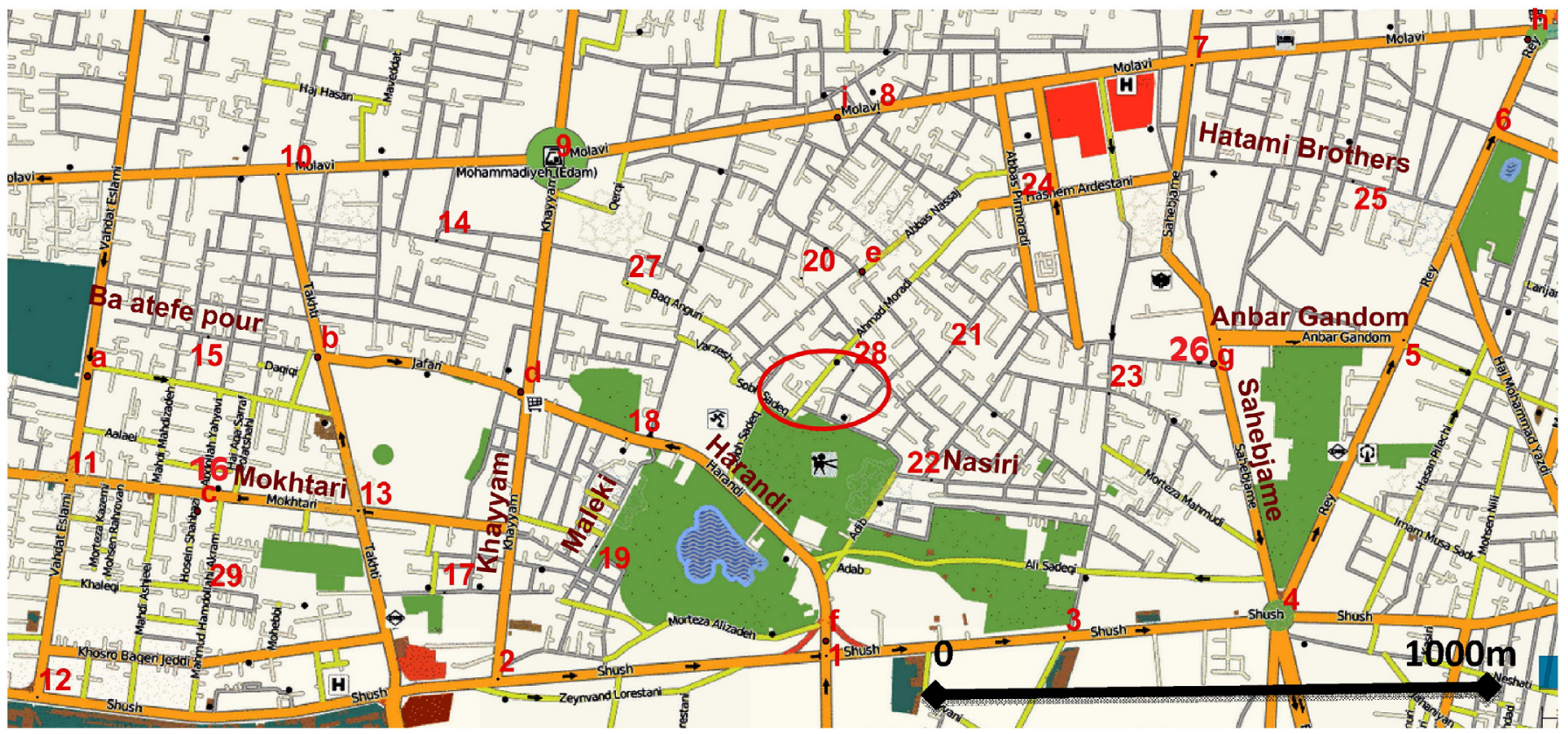

Fig. 8. The points labeled by numbers and letters are presented to the panelists to assess the permeability and legibility respectively. The oval determines the most responsive area. 


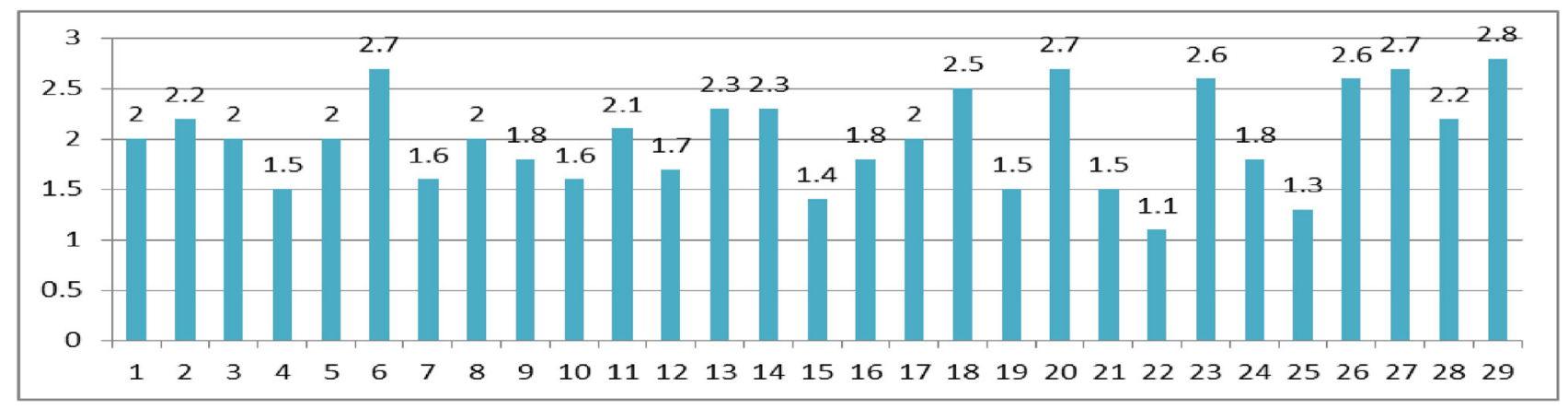

Fig. 9. The degrees of permeability for 29 evaluated points.

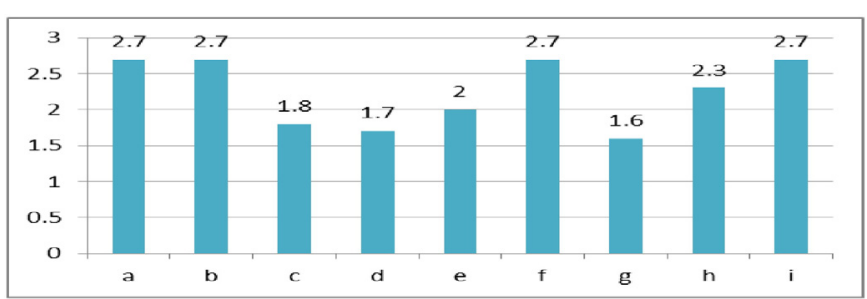

Fig. 10. The degrees of legibility for 9 evaluated neighborhoods.

Brothers" is confirmed by the patterns that emerge from Fig. 7.1 to Fig. 7.3, finally the role of the street "Ba Atefe pour" is confirmed by Fig. 7.2 and Fig. 7.3. These findings confirm that small blocks have effective impact on the permeability of the environment, which being an important property for urban design.

In order to study the notion of legibility materialized by nine parameters illustrated in Fig. 7.5 to Fig. 7.13, inhabitants were asked to express their opinion, as done for the evaluation of permeability. Fig. 11.b presents the spatial distribution of the legibility determined by people and materialized as a raster layer. The figures reveal that the spots that appear around "Mokhtari", "Khayyam" and "Sahebjame" streets (these streets are shown in Fig. 8) are confirmed by the ones of legibility shown in Fig. 7.5 to Fig. 7.13. The "Mokhtari" street is confirmed by Fig. 7.5, Fig. 7.7, Fig. 7.8, Fig. 7.9, Fig. 7.10, Fig. 7.11, Fig. 7.12 and Fig. 7.13. The "Khayyam" street is confirmed by 7.5, 7.7, 7.10, 7.11 and 7.13. The "Sahebjame" street is confirmed by 7.5, 7.6, 7.10, 7.11, 7.12 and 7.13. We can conclude that parcels with simple shape, paths with less bend and districts with more singularity have stronger effects on the legibility of environment.

The parameter "variety" is studied and verified via a land-use map of the study area (Fig. 12.a) (Tehran Municipality, 2006). It appears that the main areas shown in Fig. 7.4 as the most variegated are confirmed by the land-use variegated regions that appear in the land-use map. The pencil sketches shown in Fig. 12.a delimit the main spots in Fig. 7.4. Fig. 12.b presents a monochrome map that visually presents the boundary of extents in which the visual contrast of the layout is more. They also are the main spots as revealed by the patterns shown in Fig. 7.16.

Finally, in order to verify the parameter "robustness" a map of landuse changes has been considered. The robustness as described in Section 3.1 is the degree to which people can use a given place for different purposes and activities. This information was not officially available at the time of the study as also changing land uses in the studied region is prohibited for many years. However, we manage to find the most illegally changed areas via our field observation and domestic residents' feedbacks. In particular, the intersection between "Sahebjame", "Anbar Gandom" and "Khayyam streets" in the center of extent are the most changed areas (these streets are shown in Fig. 8). It appears that this issue is also confirmed by the dark patterns shown in Fig. 7.14 and 7.15 and the two map layers related to robustness.

\section{Summary and future works}

The research presented in this paper develops a spatial database and an ontology-based modeling approach that should contribute to explanation better understanding of the relationships between the forms and the perception of the city as revealed by the concept of responsive environments. The peculiarity of our approach lies in the combination of a domain-based ontological approach while it satisfies enough reusability and flexibility to reflect different urban modeling viewpoints. The modeling approach introduces a set of spatial metrics that qualify different properties of an urban layout, from low level to high levels primitives and from the ontological to the spatial database levels. The whole approach is implemented by a three-module
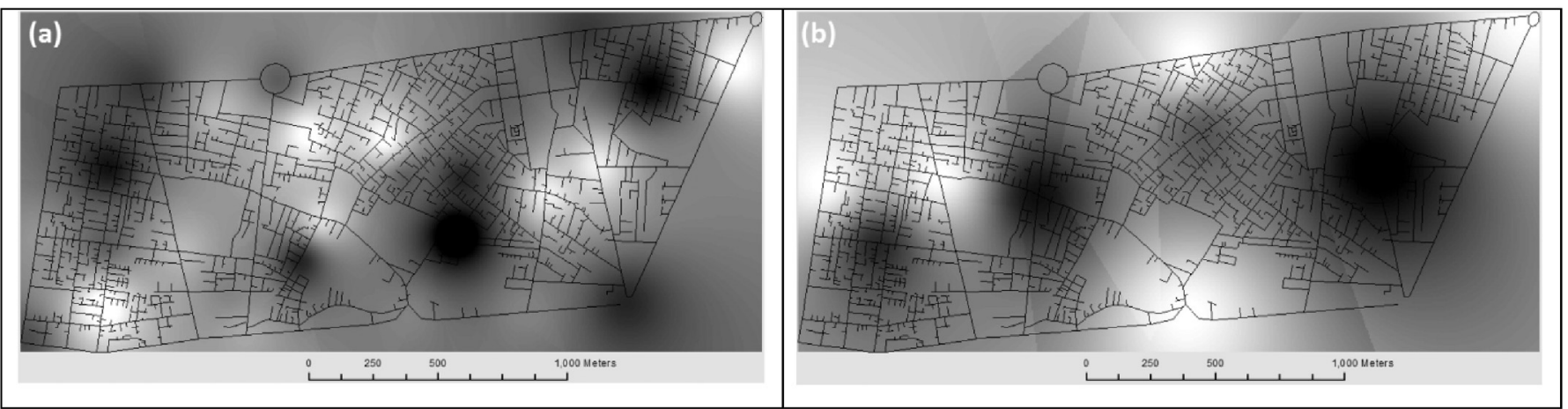

Fig. 11. (a) Distribution of permeability and (b) the legibility revealed by inhabitants' opinions. 


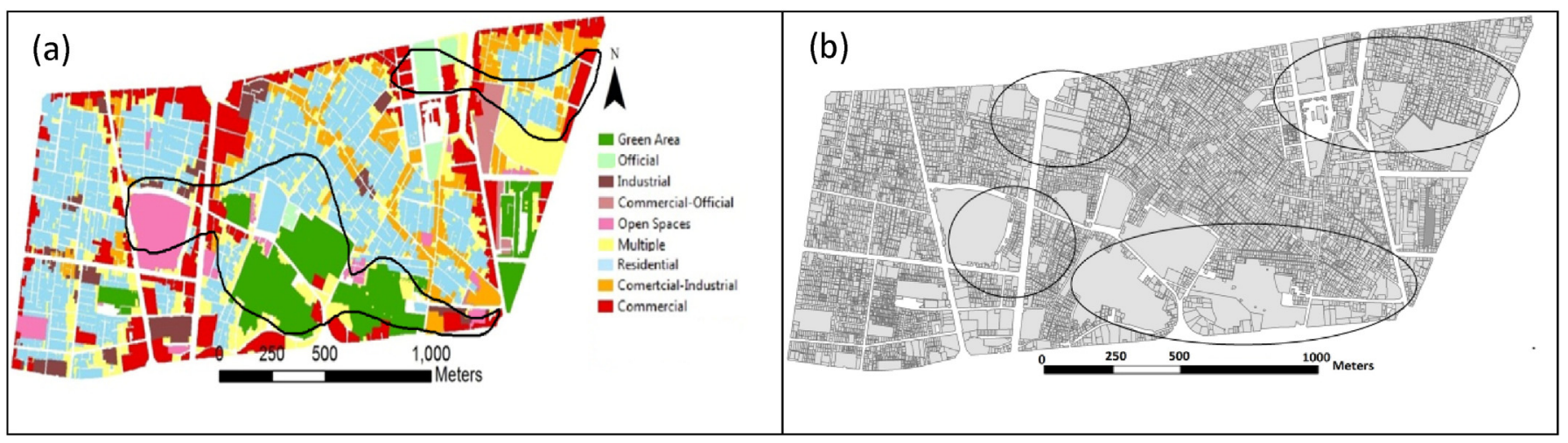

Fig. 12. (a) Land-use map in the study area. The black sketches are the spots as revealed in Fig. 7.4, (b) a monochrome map that visually presents the extents with visual contrast.

framework: urban subject, urban environment and spatial database. The developed framework is progressive as it starts from the conceptual level, to a computational representation of the elements that shape the city at different levels of abstraction, to the application to a spatial database whose potential is illustrated by a case study applied to a region in a district of the city of Tehran in Iran.

The modeling framework is based on the concepts of "Responsive Environments" previously introduced by Bentley and his colleagues. We develop a formal ontology closely associated to a conceptual representation and spatial database. These components are connected by a series of mapping rules identified as bridge rules. At the manipulation level, a series of operations derive a series of quantitative and spatially related parameters that allow an evaluation of the different responsive environment parameters identified for revealing the urban forms and properties of a given urban system.

The whole approach has been applied to a bounded region in the 12th municipal district of the city of Tehran in Iran. This area is intrinsically non responsive when perceived and navigating through, and as also inferred from the panelists selected. An important objective of our approach was to reveal the responsiveness of this area. The figures exhibited by the application of our responsive environment approach were compared to the inhabitants' opinions. Overall, it appears that the permeability, legibility, variety and robustness as derived by our modeling approach are relatively confirmed by people's opinion as well as other documents such as land-use maps and field observations. The results show the valuable role of the spatial metrics identified when studying the urban forms and opportunities and the way inhabitants perceive them.

The developed framework can provide a support for the evaluation of the different characteristics of the structural layout of an urban system via a series of spatial metrics. Urban designers might simulate different urban layouts and evaluate their impact on the legibility and robustness levels to mention some of the main properties exhibited by our modeling approach. The approach can be also applied to the different stages of an urban environment project and overall for urban studies.

Overall, the whole approach is encouraging but still deserves a refinement of the modeling principles applied and additional experiments. In particular, we plan to develop an integration of the spatial third dimension to enrich the specification of the responsive environment parameters. Also the semantic queries applied to exhibit and reveal the responsiveness of the environment are crispy evaluated. In future works, we plan to apply multivalued logics like rough sets and fuzzy sets in the procedure of semantic matching. The integration of additional semantics and criteria to represent the responsiveness of the urban environment is another avenue of research we also considering as potential valuable development of the modeling approach. The whole framework might be used not only to qualify the districts of a given city, but also to study differences and commonalities between different cities. This is another experimental evaluation we would like to explore.

\section{References}

Aguilera, F., Valenzuelaa, L. M., \& Botequilha-Leitãob, A. (2011). Landscape metrics in the analysis of urban land use patterns: A case study in a Spanish metropolitan area. Landscape and Urban Planning, 99, 226-238.

Ariza-Villaverde, A. B., Jiménez-Hornero, F. J., \& Ravé, E. G. D. (2013). Multifractal analysis of axial maps applied to the study of urban morphology. Computers, Environment and Urban Systems, 38, 1-10.

Bahrainy, H., \& Khosravi, H. (2013). The impact of urban design features and qualities on walkability and health in under-construction environments: The case of Hashtgerd New Town in Iran. Cities, 31, 17-28.

Bentley, I., Alcock, A., Murrain, P., McGlynn, S., \& Smith, G. (1985). Responsive environments: A manual for designers. London: The Architectural Press.

Bhatt, M., Hois, J., \& Kutz, O. (2011). Ontological modelling of form and function for architectural design. Applied Ontology, 1-32.

Chena, Y., Li, X., Zheng, Y., Guan, Y., \& Liu, X. (2011). Estimating the relationship between urban forms and energy consumption: A case study in the Pearl River Delta 2005-2008. Landscape and Urban Planning, 102, 33-42.

Contreras, D., Blaschke, T., Kienberger, S., \& Zeil, P. (2013). Spatial connectivity as a recovery process indicator: The L'Aquila earthquake. Technological Forecasting and Social Change, $80,1782-1803$.

Delgado, F., Mart'inez-Gonzalez, M., \& Finat, J. (2013). An evaluation of ontology matching techniques on geospatial ontologies. International Journal of Geographical Information Science, 27, 2279-2301.

Edussuriya, P., Chan, A., \& Yec, A. (2011). Urban morphology and air quality in dense residential environments in Hong Kong. Part I: District-level analysis. Atmospheric Environment, 45, 4789-4803.

Eeftens, M., Beekhuizen, J., Beelen, R., Wang, M., Vermeulen, R., Brunekreef, B., ... Hoek, G. (2013). Quantifying urban street configuration for improvements in air pollution models. Atmospheric Environment, 72, 1-9.

Fan, C., \& Myint, S. (2014). A comparison of spatial autocorrelation indices and landscape metrics in measuring urban landscape fragmentation. Landscape and Urban Planning, $121,117-128$.

Filicaia, I. G. D. (2007). The housing forms and urban morphology of poverty areas in the London borough of Islington. University College London.

Futcher, J. A., Kershawb, T., \& Mills, G. (2013). Urban form and function as building performance parameters. Building and Environment, 62, 112-123.

Gainza, X., \& Livert, F. (2013). Urban form and the environmental impact of commuting in a segregated city, Santiago de Chile. Environment and Planning B: Planning and Design, 40, 507-522.

Gruber, T. R. (1993). A translation approach to ontology specifications. Knowledge Acquisition, 5, 199-220

Guarino, N. (1998). Formal ontology and information systems. In N. Guarino (Ed.), Formal ontology in information systems (pp. 3-17). Trento, Italy: IOS Press, Amsterdam.

Gustafson, E. J. (1998). Quantifying landscape spatial pattern: What is the state of the art. Ecosystems, 1, 143-156.

Haarslev, V., \& Moller, R. (2004). RACER user's guide and reference manual. (Version 1.7.19).

Herold, M., Couclelis, H., \& Clarke, K. C. (2005). The role of spatial metrics in the analysis and modeling of urban land use change. Computers, Environment and Urban Systems, 29, 369-399.

Hillier, B., \& Hanson, J. (1984). The social logic of space. Cambridge: Cambridge University Press.

Jiang, B., \& Claramunt, C. (2004a). A structural approach to the model generalisation of an urban street network. GeoInformatica, 8, 151-171.

Jiang, B., \& Claramunt, C. (2004b). Topological analysis of urban street networks. Environmental Planning B, 31, 151-162.

Jiang, B., \& Liu, C. (2009). Street-based topological representations and analyses for predicting traffic flow in GIS. International Journal of Geographical Information, 23, 1119-1137.

Jiang, B., Claramunt, C., \& Klarqvist, B. (2000). Integration of space syntax into GIS for modelling urban spaces. International Journal of Applied Earth Observation and Geoinformation, 2, 161-171.

Jung, C., Sun, C., \& Yuan, M. (2013). An ontology-enabled framework for a geospatial problem-solving environment. Computers, Environment and Urban Systems, 38 45-57. 
Khirfan, L. (2010). Exploring isovist fields: Space and shape in architectural and urban morphology. Cities, 27, 315-325.

Koohsari, M. J., Kaczynski, A. T., Giles-Corti, B., \& Karakiewicz, J. A. (2013). Effects of access to public open spaces on walking: Is proximity enough? Landscape and Urban Planning, 117, 92-99.

Kotoulas, S., Lopez, V., Lloyd, R., Sbodio, M. L., Lecue, F., Stephenson, M., ... Aonghusa, P. M. (2014). SPUD-Semantic processing of urban data. Web Semantics: Science, Services and Agents on the World Wide Web, 24, 11-17.

Kutz, O., Lutz, C., Wolter, F., \& Zakharyaschev, M. (2004). E-connections of abstract description systems. Artificial Intelligence, 156, 1-73.

Liu, T., Luan, Y., \& Zhong, W. (2012). Earthquake responses of clusters of building structures caused by a near-field thrust fault. Soil DynamicsandEarthquakeEngineering, 42, $56-70$.

Lüscher, P., Weibel, R., \& Burghardt, D. (2009). Integrating ontological modelling and Bayesian inference for pattern classification in topographic vector data. Computers, Environment and Urban Systems, 33, 363-374.

Lutz, M., \& Klien, E. (2006). Ontology-based retrieval of geographic information. International Journal of Geographical Information Science, 20, 233-260.

Lynch, K. (1960). The image of the city. Cambridge, Massachusetts: The MIT Press.

Mata, F., \& Claramunt, C. (2011). GeoST: Geographic, thematic and temporal information retrieval from heterogeneous web data sources. In K. Tanaka, P. Frohlich, \& K. S. Kim (Eds.), 10th international symposium on web and wireless GIS (W2GIS 2010). 6574. (pp. 5-20). Kyoto, Japan: Springer.

McGarigal, K., \& Marks, B. (1995). FRAGSTATS: Spatial pattern analysis program for quantifying landscape structure. U.S. Forest Service General Technical Report PNW-GTR-351.

Moulden, B., Kingdom, F., \& Gatley, L. (1990). The standard deviation of luminance as a metric for contrast in random-dot images. Perception, 19, 79-101.
Noy, N. F., Fergerson, R. W., \& Musen, M. A. (2000). The knowledge model of Protege2000: Combining interoperability and flexibility. 2th International Conference on Knowledge Engineering and Knowledge Management (EKAW'2000). France: Springer.

Raskin, R. G., \& Pan, M. J. (2005). Knowledge representation in the semantic web for earth and environmental terminology (SWEET). Computers and Geosciences, 31, 1119-1125.

Rybarczyk, G., \& Wu, C. (2014). Examining the impact of urban morphology on bicycle mode choice. Environment and Planning B: Planning and Design, 41, 272-288.

Scheuer, S. Haase, D. \& Meyer, V. (2013). Towards a flood risk assessment ontology Knowledge integration into a multi-criteria risk assessment approach. Computers, Environment and Urban Systems, 37, 82-94.

SheikhMohammadZadeh, A., \& Rajabi, M. A. (2013). Analyzing the effects of the street network configuration on the efficiency of an urban system. Cities, 31, 285-297.

TAC (1999). In T. A. o. Canada (Ed.), Geometric design guide for Canadian roads. Canada: Transportation Association of Canada.

Tehran Municipality (2006). In U. d. a. architecture (Ed.), Developing plan for district 12 (pp. 88) (1st ed.). Tehran: Tehran Municipality.

Tomko, M., \& Winter, S. (2013). Describing the functional spatial structure of urban environments. Computers, Environment and Urban Systems, 41, 177-187.

Uschold, M., \& Gruninger, M. (1996). Ontologies: Principles, methods and applications. Knowledge Engineering Review, 11.

Voordea, T. V. d., Jacquet, W., \& Cantersa, F. (2011). Mapping form and function in urban areas: An approach based on urban metrics and continuous impervious surface data. Landscape and Urban Planning, 102, 143-155.

Yimin, C., Li, X., Zheng, Y., Guan, Y., \& Liu, X. (2011). Estimating the relationship between urban forms and energy consumption: A case study in the Pearl River Delta, 2005-2008. Landscape and Urban Planning, 102, 33-42. 